\section{Testing for AIDS}

SIR - I would like to clarify several statements in your news article on anti-HTLVIII testing of blood donors (Nature 13 December, p.583). I am director of the American Red Cross Blood Services, Northeast Region, which encompasses only Massachusetts and Maine, not "the northeastern United States" as your article indicates.

Neither this nor other regional blood services are being rushed by Secretary of Health and Human Services Margaret Heckler. It has been said, and it is possible, that there is pressure from high levels of government to expedite the availability of a test for AIDS (acquired immune deficiency syndrome) infectivity. Licensing must come before availability, and this decision lies with the the Food and Drug Administration (FDA), as you correctly pointed out. Any "rushing" would thus be intragovernmental, or between the government and test-kit manufacturer. Regional blood services will implement licensed testing if mandated by FDA. It is my hope, however, that licensing or mandating will not occur until regional blood centres are in a position to implement the test with reasonable assurance that the number of false positive test results will be minimal, and there is a clear understanding of the significance of anti-HTLV-III test positivity. To my knowledge neither issue has yet been resolved. What we really need is a reliable, quick, simple, inexpensive test for AIDS infectivity (not just presumptive evidence of previous exposure to HTLVIII). The safety of blood transfusion thus could be significantly improved.

The tests being developed have the potential for causing undue concern for a number of healthy donors while not removing from the blood supply all the units that are potentially infective for AIDS. Indeed, data have recently been presented at the American Society of Hematology meetings that indicate that apparently healthy persons can carry HTLV-III virus for significant periods without developing the antibody.

Peter L. PAge

American Red Cross Blood Services,

Northeast Region,

Needham, Massachusetts 02194, USA

\section{Thinking machines}

SIR - Further to your recent leading article on the Reith Lectures (Nature 29 November, p.397), many people, particularly those working in artificial intelligence (AI), argue that a conscious/ intelligent machine could in principle be constructed on more or less present day lines; that is, it would be a Turing-like machine, with, in Professor John Searle's phrase, a "formal program".
Let us imagine such a machine. Since the program is formal, it can be printed out, in some approximation to normal language, on paper. Now it is the essence of programming that anything the machine can do can in principle be understood in terms of the printed program. Any output(s) generated by the machine in response to any input(s) are implicit in the program, in the marks on the paper. But the Turing argument was basically that if the inputoutput exchange looks conscious/intelligent, then the machine (and therefore the program) must be regarded as conscious/ intelligent. This means that whatever consciousness/intelligence resides in the machine must also reside in these marks. But surely not even the most dedicated AI enthusiast would assert this.

While this argument is perhaps not rigorous, it does seem to me to support Professor Searle's thesis that a formally programmed machine cannot be conscious/ intelligent.

Affander 967,

J. F. CRAWFORD

\section{$\mathrm{CH}$-5303 Wuerenlingen,}

Switzerland

\section{Numerology}

SIR - In July 1983 (Nature 304, 11; 1983) I presented a series of numerological results concerning the masses of elementary particles. My article was accompanied by a sceptical commentary, in which you gave the view that the results are likely to be no more than coincidence and are in any case a "fruitless pursuit". Whether or not it is a fruitless pursuit is, in my view, still very much open to debate, although I doubt whether any such debate would reveal anything other than a wide variety of subjective opinion on the matter. But the real issue is, or should be, whether or not the results can be dismissed as no more than coincidence. As such, the crucial factor is the statistical evidence.

Among the results in my article were two relating to the combined mass of the particles in the basic meson octet, and the basic baryon octet, relative to the proton mass. The combined mass of the particles $\pi^{0}, \pi^{+}, \pi^{-}, \mathrm{K}^{+}, \mathrm{K}^{-}, \mathrm{K}^{0}, \mathrm{~K}^{0}$, and $\eta$, of the basic meson octet, is 3.13935 times the proton mass. The multiplier here is close to $\pi$, or 3.1415926 . Such a close relationship clearly has a specific chance of having arisen. 3.13935 is $\pi$ to the power of 0.999376, and a little elementary mathematics shows that only one random rational number in 801.2 will have such accuracy relative to any integral power of $\pi$.

The combined mass of the particles $\varrho, \eta$, $\Lambda, \Sigma^{+}, \Sigma^{0}, \Sigma^{-}, \Xi^{0}$, and $\Xi^{-}$, of the basic baryon octet, is 9.8146 times the proton mass. The multiplier here is close to $\pi^{2}$, or 9.869604. Once again, the relationship had a specific chance of occurring. 9.8146 is $\pi$ to the power of 1.995118 , so only one randam rational number in 102.4 will have such accuracy relative to any integral power of $\pi$. This means that the odds are 82,000 to one against any pair of random rational numbers occurring with an overall accuracy, relative to integral powers of $\pi$, similar to that of the above two results.

Given the simple nature of the results, it seems clear that coincidence is unlikely (though not impossible). Here we have a phenomenon that deserves serious scientific attention, and not a casual dismissal in terms of coincidence. Furthermore, in view of the absence of any full physical theory of particle mass, there ought to be greater attention given to those observations that might provide useful clues pointing in the right direction. Can science really afford to ignore such results?

Flat 2,

Peter Stanbury

3 Ferndale,

Tunbridge Wells, Kent TN2 3RL, UK

\section{Back to Kekulé}

SIR - In his Commentary on the philosophy of science, George Gale ${ }^{1}$ mentioned that the dialogue between physicists and philosophers of science had recently been augmented by computer scientists and theoretical biologists.

Even more recently, some practitioners of chemistry have also joined in the dialogue: philosophical aspects of surface chemistry ${ }^{2}$, physical organic chemistry ${ }^{3}$, bio-organic chemistry ${ }^{4,5}$ and quantum chemistry ${ }^{6}$ have been discussed in the literature.

Kekulé $^{7}$ published some thoughts in 1878 , and that address is still worth reading today.

\section{Bradford and Ilkley}

F.M. AKEROYD

Community College,

Great Horton Road,

Bradford, West Yorkshire, BD7 IAY, UK

1. Gale, G. Nature 312, 491 (1984).

2. Good, R.J. Chem Technol. 10, 100 (1980)

3. Zollinger, H. Chem. Br. 16, 257 (1980).

4. Akeroyd, F.M. J. Chem. Educ. 61, 697 (1984)

5. Akeroyd, F.M. J. Chem. Educ. 61, 434 (1984).

6. Weininger, S.J. J. Chem. Educ. 61, 939 (1984).

7. Kekulé, A. Nature 18, 210 (1878).

\section{Human embryos}

SIR - I support the views expressed by John R. Ling (Nature 24 January, p.262). As a biologist, I can see no justification for an arbitrary age below which experimentation on human embryos is legitimate, while above this level it is not. Surely the zygote becomes a potential human being from the moment of fertilization and, if so, it is from that moment entitled to as much respect for, and protection of, its life as is accorded by most societies to a child or adult. In this respect, I agree strongly with the minority of the Warnock committee, and it will be interesting to learn the views of biologists and others among the readership of Nature.

J.R. BAKER

4 Belvoir Road,

Cambridge CB4 1JJ, UK 